\title{
Highly efficient gene transfer to solid tumors in vivo by tumor-selective replicating retrovirus vectors
}

\author{
YIN-CHE LU ${ }^{1}$, YI-PING LUO ${ }^{2}$, YU-WEN WANG ${ }^{2}$ and CHIEN-KUO TAI ${ }^{2}$ \\ ${ }^{1}$ Division of Hematology-Oncology, Department of Internal Medicine, Chia-Yi Christian Hospital, Chia-Yi; \\ ${ }^{2}$ Department of Life Science and Institutes of Molecular Biology and Biomedical Science, \\ National Chung Cheng University, Min-Hsiung, Chia-Yi, Taiwan, R.O.C.
}

Received December 7, 2009; Accepted January 15, 2010

DOI: 10.3892/ijmm_00000403

\begin{abstract}
Tumor-selective replicating viruses are attractive tools for cancer gene therapy, but generally achieve only transitory tumor suppression. However, replicating retrovirus vectors (RRVs) can achieve highly efficient and tumorselective transduction, as well as persistent expression of transgenes. We therefore developed RRVs that express the yeast cytosine deaminase (yCD) and herpes simplex virus thymidine kinase (TK), which exhibit remarkably enhanced cytotoxicity after administration of the prodrugs 5-fluorocytosine (5-FC) and ganciclovir (GCV) concomitant with the efficiency of their replicative spread, and tested their therapeutic effect in vitro and in vivo. In subcutaneous MDAMB-435 human breast cancer xenograft models, RRVmediated yCD and TK suicide gene therapy significantly suppressed tumor growth after prodrug administration. Notably, no systemic spread of the vector to extratumoral tissues was detected. Our results thus demonstrate that efficient, tumor-selective, and stable integration achieved by RRVs causes efficient cell killing upon prodrug administration, resulting in significant suppression of tumor growth.
\end{abstract}

\section{Introduction}

Vectors derived from retroviruses have been widely applied for gene transfer to target cells in vitro and in vivo, and in fact retroviruses are used in the majority of gene therapy trials to date (1). Retrovirus vectors, based on murine leukemia virus (MLV), possess several distinct inherent advantages in cancer gene therapy: achieving transduction only in actively dividing cells (2-4); stable expression of desired transgenes by

Correspondence to: Dr Chien-Kuo Tai, Department of Life Science, National Chung Cheng University, 168 University Road, Min-Hsiung, Chia-Yi 621, Taiwan, R.O.C.

E-mail: biockt@ccu.edu.tw

Key words: replicating retrovirus, cytosine deaminase, thymidine kinase, suicide gene, breast cancer integration of viral genome into chromosomes of target cells to offer the potential for long-term cure; and low immunogenicity compared to some other virus vectors (5-8). Conventional retrovirus vectors are replication-defective retrovirus (RDR) vectors in which the viral genes gag, pol, and env are removed to develop a safer gene delivery carrier. Despite the fact that RDR vectors have been applied for a number of cancer gene therapy clinical trials, the low efficiency of gene transfer in solid tumors exhibited by RDR vectors becomes their major limitation for cancer gene therapy (9-11).

In order to improve transduction efficiency of retrovirus vectors, we have developed replicating retrovirus vectors (RRVs) derived from MLV (12-14). The RRVs retain a fully native viral genome and thus possess the ability to replicate. In addition to an intact MLV genome including long terminal repeat (LTR), packaging signal ( $\Psi)$, and genes gag, pol and env, the RRVs contain an internal ribosome entry site (IRES)transgene cassette between the env gene and the $3^{\prime}$ untranslated region (3' UTR). While the RRVs infect a target tumor cell, each transduced cell itself becomes a virus-producing cell to sustain further transduction events, thus greatly increasing transduction efficiency in vivo.

To evaluate its potential as a therapeutic vector for the treatment of cancer, we incorporated into RRVs the yeast cytosine deaminase (yCD) and herpes simplex virus thymidine kinase (TK) suicide genes. The yCD deaminates the nontoxic prodrug 5-fluorocytosine (5-FC) to the highly toxic metabolite 5-fluorouracil (5-FU) (15), which is also one of the most active antineoplastic agents in conventional cancer chemotherapy. The TK efficiently monophosphorylates ganciclovir (GCV), a guanosine analog, which is subsequently converted into GCVtriphosphate by endogenous mammalian kinases $(16,17)$. Cellular DNA polymerase further incorporates the GCVtriphosphate into replicating DNA, thereby arresting DNA replication and causing cell death (18).

In this study, we explored the use of RRVs for suicide gene therapy of breast tumors. We examined the survival of transduced tumor cells in vitro as well as tumor growth in vivo after inoculation of RRV followed by treatment with their respective prodrugs. We evaluated whether the significantly higher transduction levels achieved by RRV have the potential to greatly enhance the efficacy of suicide gene therapy for the treatment of breast tumors. 


\begin{tabular}{|c|c|c|c|c|c|c|}
\hline ACE-GFP & \begin{tabular}{|l|l|l}
$R$ & \\
\end{tabular} & gag & pol & $e n v$ & IRES-GFP & \begin{tabular}{l|l} 
U3 & $R$ \\
\end{tabular} \\
\hline ACE-TK & $\mathrm{R}$ & gag & pol & $e n v$ & IRES-TK & \begin{tabular}{l|l} 
U3 & $R$ \\
\end{tabular} \\
\hline GS4-GFP & & gag & pol & env & IRES-GFP & \begin{tabular}{l|l} 
U3 & $R$ \\
\end{tabular} \\
\hline GS4-dsRed & & gag & pol & env & IRES-dsRed & \begin{tabular}{l|l} 
U3 & $R$ \\
\end{tabular} \\
\hline GS4-yCD & us & gag & pol & env & IRES-yCD & \begin{tabular}{l|l}
$\mathrm{U} 3$ & $\mathrm{R}$ \\
\end{tabular} \\
\hline
\end{tabular}

Figure 1. Schematic representation of replicating retrovirus vectors. ACE-GFP and ACE-TK are replication-competent amphotropic MLV vectors containing transgene positioned between amphotropic env and 3' UTR. GS4-GFP, GS4-dsRed, and GS4-yCD are replication-competent GALV vectors containing transgene. Unshaded regions represent sequences derived from MLV and grey shaded regions represent those derived from GALV. IRES, internal ribosome entry site; $\Psi$, packaging signal.

\section{Materials and methods}

Construction of retrovirus vectors. Plasmid pACE-GFP (13) is a replication-competent amphotropic MLV vector containing an IRES-GFP sequence positioned between amphotropic env and 3' UTR. The TK gene was amplified from the plasmid pFRA-TK (19) by PCR using Pfu polymerase (Stratagene, La Jolla, CA, USA), and used to replace the GFP sequence in pACE-GFP, generating plasmid pACETK. Plasmid pGS4-GFP (20) is a replication-competent gibbon ape leukemia virus (GALV) vector containing an IRES-GFP positioned between the env gene and 3' UTR. The yCD gene sequence was amplified from yeast genome by PCR and was used to replace the GFP sequence in pGS4-GFP, generating plasmid pGS4-yCD.

Cell lines and virus vector production. 293T, MDA-MB-435, and MCF7 cells were cultured in Dulbecco's modified Eagle's medium (DMEM) with $10 \%$ fetal bovine serum (FBS) (Invitrogen, Carlsbad, CA, USA). T47D cells were cultured in DMEM with $10 \% \mathrm{FBS}, 1.5 \mathrm{~g} / 1$ sodium bicarbonate, $1 \mathrm{mM}$ sodium pyruvate, and $10 \mu \mathrm{g} / \mathrm{ml}$ bovine insulin (Sigma, St. Louis, MO, USA). Virus particles were produced by transient transfection of the RRV-encoding plasmids into 293T cells using calcium phosphate precipitation as described previously (21). The virus-containing supernatant was collected 2 days post-transfection, filtered through a $0.45-\mu \mathrm{m}$ filter, and frozen at $-80^{\circ} \mathrm{C}$ for later use. For titer determination, viral supernatant was added to $\sim 20 \%$ confluent tumor cells in the presence of $4 \mu \mathrm{g} / \mathrm{ml}$ polybrene (Sigma) in six-well plates. Viral supernatant was then removed $24 \mathrm{~h}$ post-infection, and cells were incubated in regular media with $25 \mu \mathrm{M}$ AZT (Sigma) for $48 \mathrm{~h}$ and were analyzed for GFP and dsRed expression using a FACScan (Becton Dickinson, Franklin Lakes, NJ, USA). Retrovirus titer was represented as transducing units per milliliter (TU/ml).

Transduction of tumor cells. MDA-MB-435, MCF7, and T47D cells seeded on six-well plates were infected with virus ACEGFP at a multiplicity of infection (MOI) of 0.05 in the presence of $4 \mu \mathrm{g} / \mathrm{ml}$ polybrene. At various time points post-infection, the cells were analyzed for GFP expression using flow cytometry. In a separate experiment, MDA-MB-435 cells infected with ACE-GFP or GS4-GFP were mixed with uninfected cells at a ratio of $5 \%$ of the cell population and seeded onto six-well plates. At various times after mixing, the total cell population was analyzed for GFP expression by flow cytometry in order to estimate the spread of vectors.

In vitro cytotoxicity of $y C D / 5-F C$ and $T K / G C V$. Uninfected and GS4-yCD-infected MDA-MB-435 cells were mixed at various initial ratios as indicated (Fig. 3A) and seeded onto replicate 96 -well plates $\left(2 \times 10^{3}\right.$ cells per well). After overnight culture, the mixed cell populations were exposed to $1 \mathrm{mM}$ 5 -FC or to control medium without 5-FC, and viable cells were determined daily by MTS assay using the CellTiter Aqueous One Solution Cell Proliferation Assay kit (Promega, Madison, WI, USA). Similarly, uninfected and ACE-TKinfected MDA-MB-435 cells were mixed at various initial ratios as indicated (Fig. 3B) and were exposed to $10 \mu \mathrm{g} / \mathrm{ml}$ GCV or to regular medium, and viable cells were determined by MTS assay.

Vector spread in subcutaneous tumor models. For the subcutaneous breast tumor model, BALB/c nude mice (National Laboratory Animal Center, Taiwan) were subcutaneously inoculated with cell suspensions $\left(5 \times 10^{6}\right)$ consisting of ACE-GFP-transduced MDA-MB-435 mixing with uninfected MDA-MB-435 at a ratio of $1 \%$ of the cell population. Four and six weeks after tumor implantation, tumors were dissected, washed with cold Hank's balanced salt solution, minced into small pieces, and digested with $0.2 \%$ collagenase/dispase (Roche Diagnostics Corporation, Indianapolis, IN, USA). The dissociated cells were spun down, resuspended in PBS, and passed through a $100-\mu \mathrm{m}$ cell strainer (Becton Dickinson). The cells were immediately subjected to flow cytometry for GFP expression analysis. In a second experiment, nude mice were subcutaneously inoculated with cell suspensions $\left(5 \times 10^{6}\right)$ consisting of ACEGFP transduced or GS4-GFP transduced MDA-MB-435 mixing with uninfected MDA-MB-435 at a ratio of $5 \%$ of the cell population. Four weeks after tumor implantation, tumor cells were harvested as previously described and were subjected to flow cytometric analysis. 
A

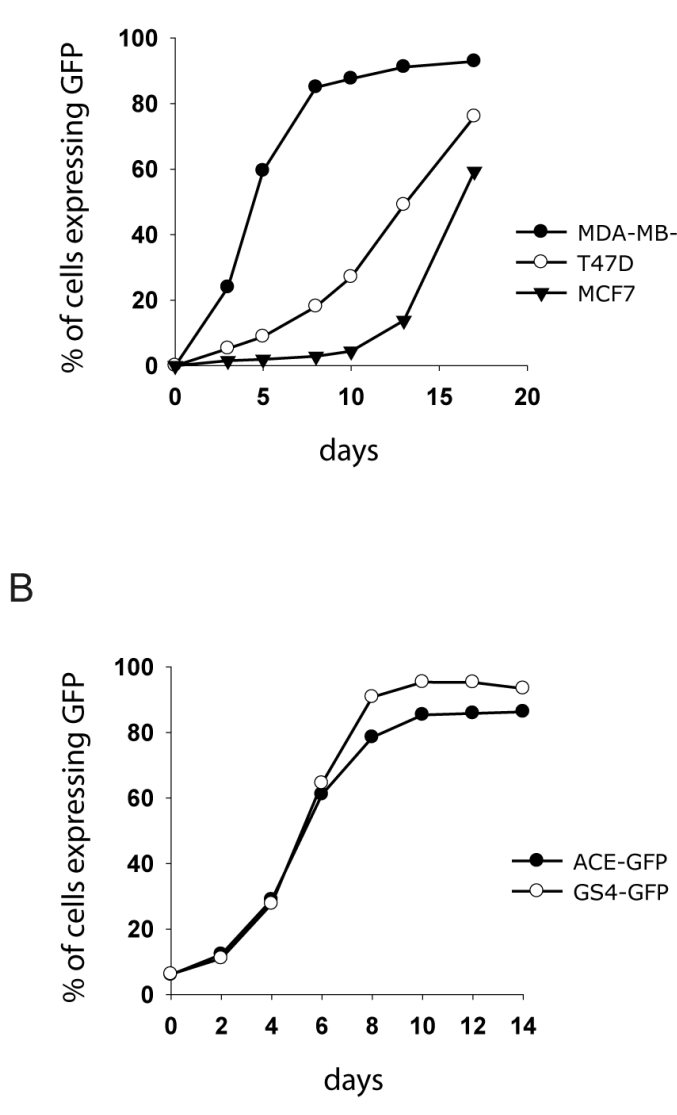

C

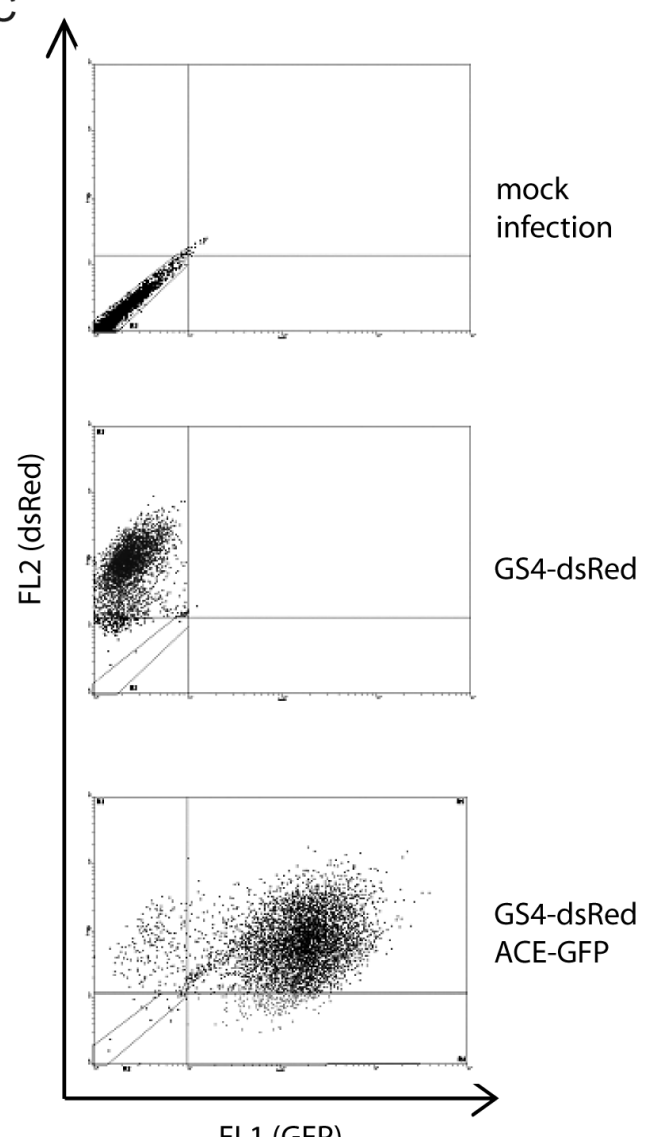

Figure 2. (A) Replication kinetics of ACE-GFP in breast tumor cells. Human breast tumor cells MDA-MB-435, MCF7, and T47D were inoculated with ACEGFP at an MOI of 0.05. At various time points after infection, the cells were analyzed for GFP expression by flow cytometry. (B) Replicative spread of RRVs in MDA-MB-435. ACE-GFP transduced or GS4-GFP transduced MDA-MB-435 cells were mixed with uninfected MDA-MB-435 at a ratio of 5\% of the cell population and seeded onto culture plates. Various times after mixing, the total cell population was analyzed for GFP expression by flow cytometry. (C) Coinfections of MDA-MB-435 with GS4-dsRed and ACE-GFP. GS4-dsRed-transduced MDA-MB-435 cells were inoculated with ACE-GFP and viral transduction was checked by flow cytometry. X-axis (FL1): GFP fluorescence; Y-axis (FL2): dsRed fluorescence.

In vivo therapeutic efficacy of $y C D / 5-F C$ and $T K / G C V$. Ten $\mathrm{BALB} / \mathrm{c}$ nude mice were inoculated with $5 \times 10^{6} \mathrm{MDA}-\mathrm{MB}-$ 435 cells pretransduced with GS4-yCD into the right dorsal flank. Seventeen days after tumor cell implantation, five mice received intraperitoneal injections of 5 -FC $(500 \mathrm{mg} / \mathrm{kg})$, while the remaining five mice received PBS injections, once daily, for a total of 10 treatments. In a second experiment, nude mice were subcutaneously inoculated with cell suspensions $\left(5 \times 10^{6}\right)$ consisting of GS4-yCD-transduced and ACE-TKtransduced MDA-MB-435 mixing with uninfected MDAMB-435 at a ratio of $5 \%$ of the cell population $(2.5 \%$ GS4yCD-transduced; $2.5 \%$ ACE-TK-transduced). When the tumors were $\sim 400 \mathrm{~mm}^{3}$ in size, mice in the uninfected group and in the group of $2.5 \% \mathrm{yCD}+2.5 \% \mathrm{TK}$ received daily injections of 5 -FC ( $500 \mathrm{mg} / \mathrm{kg}$ ) for 7 days, followed by daily injections of GCV (30 mg/kg) for 7 days.

Real-time PCR. To detect any integrated RRV sequences in genomes, real-time PCR was performed (using the ABI Prism 7700 sequence detector), targeting the 4070A amphotropic (22) and GALV envelope genes. The primers were designed to target the 4070A envelope (forward: 5'-ACCCT CAACCTCCCCTACAAG-3'; reverse: 5'-GTTAAGCGCC TGATAGGCTC-3') and GALV envelope (forward: 5'-CCT
ATTACTCCTCCTTCTGTTG-3'; reverse: 5'-GGGCCTGA TATTTTTGTCTAAG-3'). Apolipoprotein B gene for precise amounts of input genomic DNA were also quantified as an internal control (forward: 5'-CACGTGGGCTCCAGCATT-3'; reverse: 5'-TCACCAGTCATTTCTGCCTTTG-3'). Real-time PCR was done in $25 \mu \mathrm{l}$ of reaction mixture containing genomic DNA, $12.5 \mu 1$ of $2 \mathrm{X}$ SYBR green real-time PCR master mix (Toyobo, Osaka, Japan) and $300 \mathrm{nM}$ of each primer. Each sample was analyzed in triplicate, using $15 \mathrm{ng}$ of genomic DNA in each reaction. Products were amplified by 45 cycles of successive incubation at $95^{\circ} \mathrm{C}$ for $15 \mathrm{sec}$ and at $60^{\circ} \mathrm{C}$ for $1 \mathrm{~min}$. A standard curve for RRV copy number was generated by amplification of serially diluted RRV plasmid at specific copy numbers mixed into genomic DNA from spleen cells of uninfected mice.

\section{Results}

High efficiency transduction of breast tumor cells by RRVs. We have previously described the construction of ACE-GFP vector, an RRV based on amphotropic MLV containing an IRES-GFP expression cassette (Fig. 1) (13). To test the efficacy of ACE-GFP to transduce human breast tumor cells in culture, we infected MDA-MB-435, T47D, MCF7 with 
A

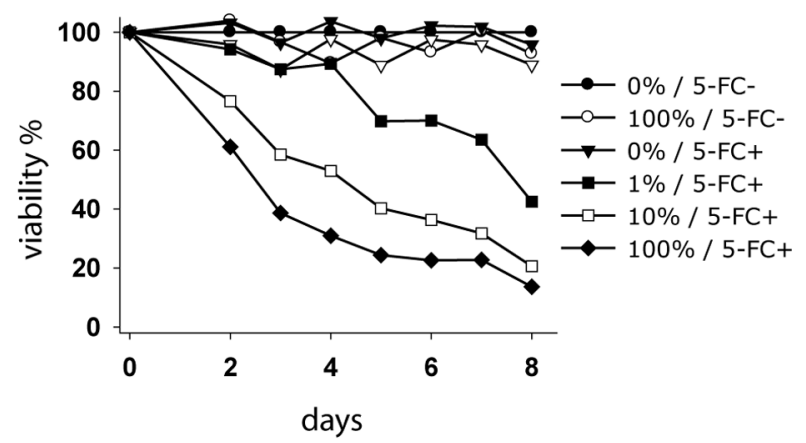

B

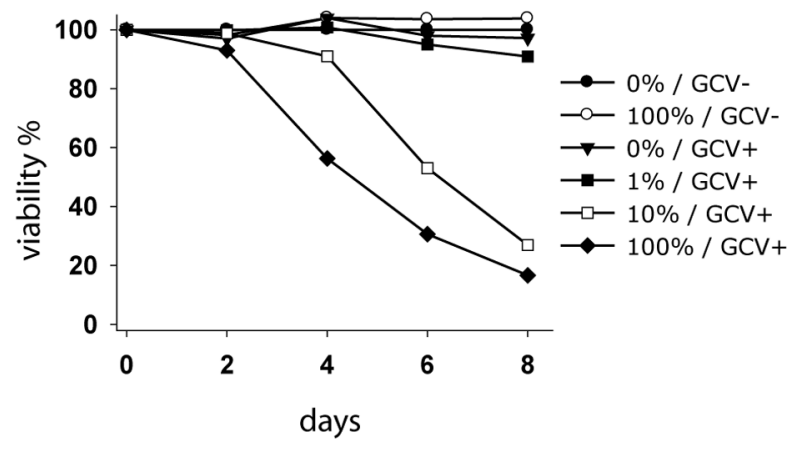

C

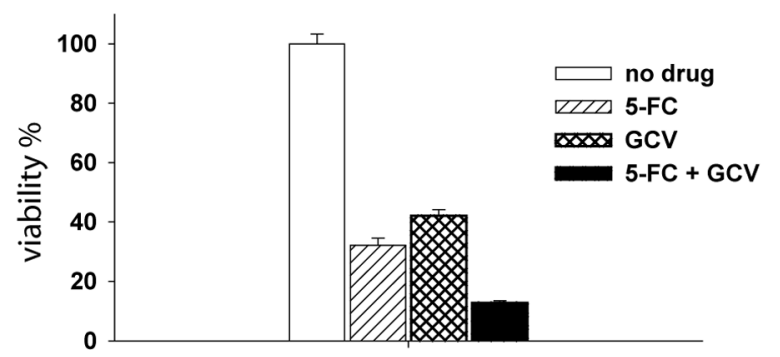

Figure 3. In vitro cytotoxicity achieved by RRVs plus prodrugs. (A) The GS4-yCD transduced and untransduced MDA-MB-435 cells were mixed at various initial ratios $(0,1,10$, and $100 \%$ of GS4-yCD transduced cells) and seeded onto 96-well plates. The mixed cell populations were exposed to $1 \mathrm{mM}$ 5-FC, and viability of cells was determined daily by MTS assay. The values shown of viability percentage of the cells were normalized to that of negative control $\left(0 \% / 5-\mathrm{FC}^{-}\right)$. 5-FC': without 5 -FC incubation. $5-\mathrm{FC}^{+}$: with 5 -FC incubation. (B) The ACE-TK transduced and untransduced MDA-MB-435 cells were mixed at various initial ratios and seeded onto 96-well plates. The mixed cell populations were exposed to $10 \mu \mathrm{g} / \mathrm{ml} \mathrm{GCV}$, and viability of cells was determined by MTS assay. (C) MDA-MB-435 cells co-transduced by GS4-yCD and ACE-TK were treated with 5-FC, GCV, or both for 5 days. Viability of cells was determined in a triplicate repeat with the MTS assay.

ACE-GFP at an MOI of 0.05 and checked viral transduction by flow cytometry. Our results showed that ACE-GFP can efficiently transduce each breast tumor cell line and spread throughout entire cell populations (Fig. 2A). We further evaluated the replication kinetics of ACE-GFP and GS4-GFP by preparing a cell mixture in which MDA-MB-435 infected with ACE-GFP or GS4-GFP were mixed with uninfected MDA-MB-435 at a ratio of $5 \%$ of the cell population and were seeded onto six-well plates. Flow cytometric analysis showed that both vectors could efficiently transfer the GFP gene to MDA-MB-435 and spread throughout entire cell populations (Fig. 2B). To study whether GS4 and ACE vectors could co-infect MDA-MB-435, we infected GS4-dsRedtransduced MDA-MB-435 with ACE-GFP at an MOI of 0.05 and checked viral transduction by flow cytometry. Images of flow cytometric analysis show that GS4-dsRed-transduced MDA-MB-435 infected with ACE-GFP eventually expressed both of dsRed and GFP, indicating that the cells could be co-infected with GS and ACE vectors (Fig. 2C).

Cytotoxic efficacy of RRV-mediated suicide gene therapy in vitro. GS4-yCD-transduced MDA-MB-435, mixed with uninfected MDA-MB-435 at varying percentages, were or were not treated with 5-FC, and viability of the cells was normalized to that of a negative control composed of uninfected MDA-MB-435 without 5-FC. When even as few as $1 \%$ of the MDA-MB- 435 cells in culture initially expressed yCD, substantial cell death was observed over time after incubation with 5-FC (Fig. 3A). The kinetics and efficiency of cell killing was highly dependent on the initial fraction of cells in the culture expressing $\mathrm{yCD}$, as higher percentages of transduced cells in the culture achieved correspondingly faster clearance of the tumor cell population and correlated with the kinetics of viral replication. To examine the cytotoxic efficacy of ACE-TK, we treated ACE-TK-transduced MDA-MB-435 mixed with uninfected cells at varying percentages with prodrug GCV. Similarly, we observed that ACE-TK/GCV caused substantial cell death when $10 \%$ and $100 \%$ of the MDA-MB-435 tumor cells initially expressed TK (Fig. 3B). To determine whether co-infection with GS4-yCD and ACETK could further enhance tumor cell killing, we treated MDA-MB-435 cells co-transduced by GS4-yCD and ACETK with 5-FC, GCV, or both for 5 days. Our result shows that when cells were treated with both prodrugs the highest level of cell killing was observed, indicating that dual-vector gene therapy achieved better cytotoxic efficacy than singlevector gene therapy (Fig. 3C).

Efficient and progressive spread of RRVs in MDA-MB-435 subcutaneous tumors. To determine the ability of RRVs to achieve efficient transduction in solid tumors in vivo, we then tested RRV in a subcutaneous MDA-MB-435 tumor model. A cell suspension consisting of ACE-GFP-transduced MDAMB-435 mixed with uninfected MDA-MB-435 at a ratio of $1 \%$ of the cell population was injected into the right dorsal flank of nude mice. The spread of RRVs in tumors was examined by quantification of GFP expression at various time points after tumor implantation. The percentage of GFPpositive cells in tumors was $22.1 \%$ at 4 weeks and $42.5 \%$ at 6 weeks after tumor implantation (Fig. 4A). These results demonstrate that ACE-GFP is capable of efficient and progressive transduction of the MDA-MB-435 solid tumor in vivo. To compare the spreading efficiency of ACE and GS4 vectors in vivo, we prepared a cell mixture in which MDA-MB-435 infected with ACE-GFP or GS4-GFP were mixed with uninfected MDA-MB-435 at a ratio of 5\% of the cell population and were implanted to right dorsal flank of nude mice. Through flow cytometric analysis of dissected tumors, the percentage of GFP-positive cells was determined 
A
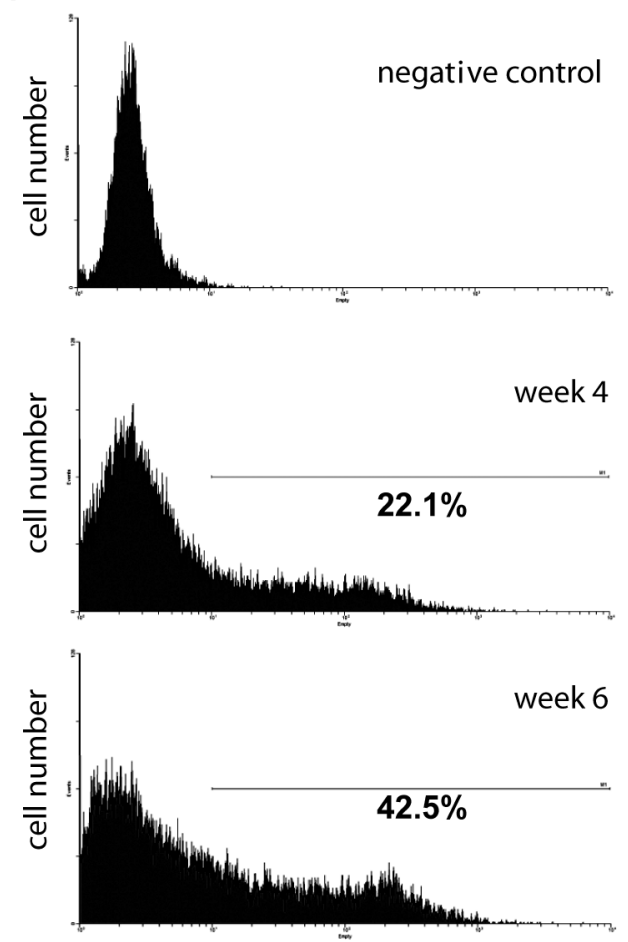

fluorescence intensity
B
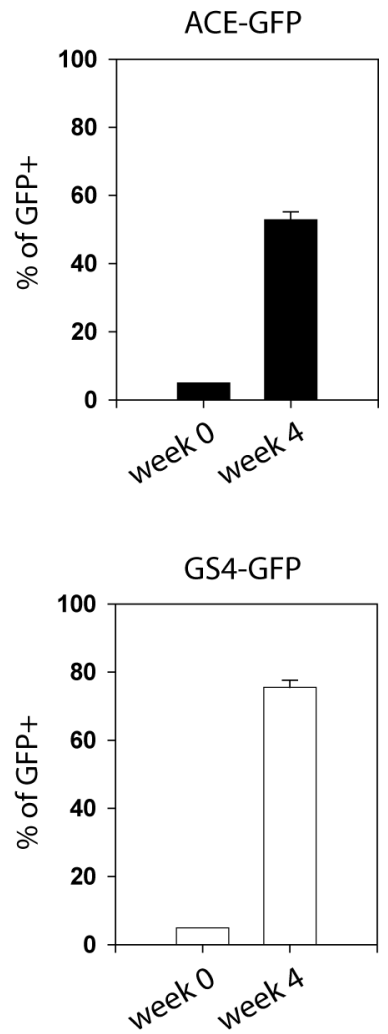

Figure 4. Spread of RRVs through solid tumors in mice. (A) A cell suspension consisting of ACE-GFP transduced MDA-MB-435 mixed with uninfected cells at a ratio of $1 \%$ of the cell population was injected into the right dorsal flank of nude mice. The spread of RRVs in tumors was examined by quantification of GFP expression after tumor implantation. The percentage of GFP-positive cells in tumors was $22.1 \%$ at 4 weeks and $42.5 \%$ at 6 weeks after tumor implantation. (B) A cell suspension consisting of ACE-GFP or GS4-GFP transduced MDA-MB-435 mixed with uninfected cells at a ratio of 5\% of the cell population was injected into the right dorsal flank of nude mice. Through flow cytometric analysis of dissected tumors, the percentage of GFP-positive cells was determined to be $53.0 \pm 2.3 \%$ (ACE-GFP) and $75.6 \pm 2.9 \%$ (GS4-GFP) at 4 weeks after implantation of tumors.

to be $53.0 \pm 2.3 \%$ (ACE-GFP) and $75.6 \pm 2.9 \%$ (GS4-GFP) at 4 weeks after implantation of tumors (Fig. 4B).

RRVs suppress the growth of MDA-MB-435 tumors after treatment with prodrugs. To evaluate any inhibitory effect of $\mathrm{yCD} / 5-\mathrm{FC}$ on tumor progression, mice bearing GS4-yCD pretransduced subcutaneous tumors were treated with PBS or 5-FC. The control group (100\% yCD/PBS) showed progressive tumor enlargement, and in contrast, the group treated with GS4-yCD plus 5-FC (100\% yCD/5-FC) showed progressive tumor shrinkage (Fig. 5A). Next, to determine whether a lower ratio of tumor cells expressing RRV could suppress tumor growth, we treated mice bearing subcutaneous MDAMB-435 tumors in which 5\% (2.5\% GS4-yCD-transduced; $2.5 \%$ ACE-TK-transduced) of the cells initially expressed RRVs with prodrugs 5-FC and GCV. GS4-yCD+ACE-TK/ $5-\mathrm{FC}+\mathrm{GCV}$ treatment resulted in significant suppression of tumor growth, compared with the control group (neg/ 5-FC+GCV) (Fig. 5B).

Biodistribution of RRVs during prolonged replication in animals. To estimate the biosafety of RRV for gene therapy in vivo, we did a quantitative real-time PCR analysis of genomic DNA extracted from tumors and extratumoral organs using primers specific for the 4070A amphotropic envelope and GALV envelope. Mouse apolipoprotein B gene for precise amounts of input genomic DNA were also quantified as an internal control. As expected, RRV was readily detected in the GS4-yCD and ACE-TK transduced MDA-MB-435 tumors by PCR amplification of both envelope sequences. However, no detectable RRV signals were observed in genomic DNA from extratumoral organs including heart, liver, spleen and bone marrow in the same animals (data not shown).

\section{Discussion}

This study demonstrates that RRVs can efficiently transduce and stably propagate in human tumor cells in vitro, and can achieve high transduction efficiency in vivo compared to conventional RDR vectors previously employed in clinical trials (10). In vitro cytotoxicity studies showed that substantial tumor cell killing can be achieved when initially even as few as $1 \%$ of the tumor cells were producing RRVs, indicating that the highly efficient gene transfer by progressive replicative spread from the initial inoculum greatly increased suicide gene toxicity. Flow cytometric analyses and PCR analyses demonstrated that RRV replication in vivo was efficient and was highly restricted to the tumor tissue itself, suggesting that the absolute requirement for cell division results in a high degree of tumor selectivity. While the possibility that the RRV might transduce non-cancerous but actively replicating cells, such as neovascular endothelial cells, requires further careful examination, the incorporation of suicide genes into the RRV 


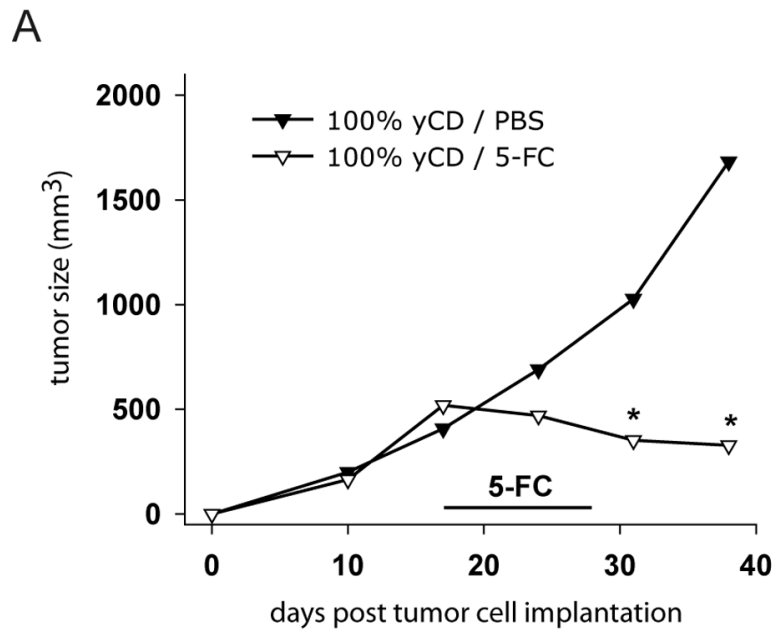

B

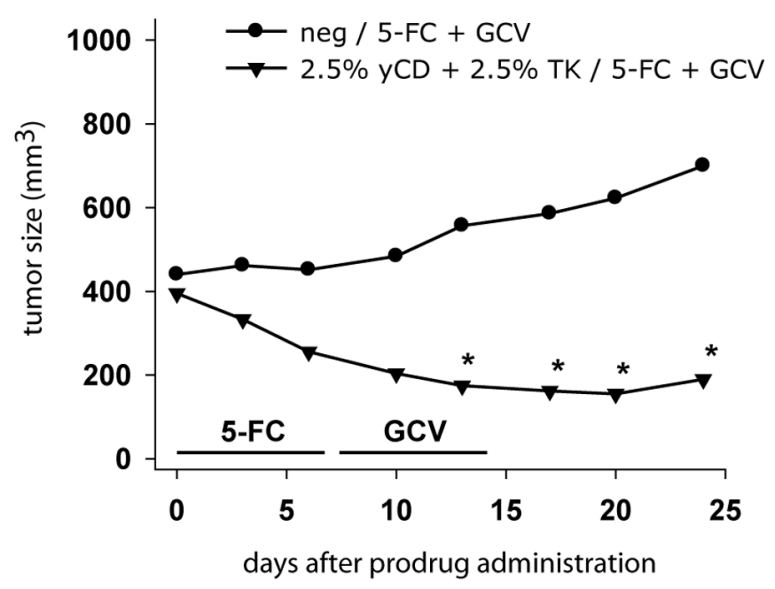

Figure 5. Anti-tumor effect achieved by RRV suicide gene therapy. (A) Growth of GS4-yCD pretransduced tumor in response to 5-FC treatment. Mice bearing GS4-yCD transduced MDA-MB-435 xenografts were treated with PBS or 5-FC for a period of 10 days from the 17th day post-tumor cell implantation. Statistically significant differences in tumor size between the GS4-yCD/5-FC-treated group $(n=5)$ and the control group $(n=5)$ were observed. ${ }^{*} \mathrm{P}<0.05$. (B) Growth of tumor in response to GS4-yCD and ACETK plus 5-FC and GCV treatments. Mice bearing subcutaneous MDA-MB435 tumors in which $5 \%$ (2.5\% GS4-yCD-transduced; $2.5 \%$ ACE-TKtransduced) of the cells initially expressed RRV were treated with 5-FC and GCV. Statistically significant differences in tumor size between the GS4$\mathrm{yCD}+\mathrm{ACE}-\mathrm{TK} / 5-\mathrm{FC}+\mathrm{GCV}$ treated group $(\mathrm{n}=8)$ and the control group $(\mathrm{n}=6)$ were observed. ${ }^{*} \mathrm{P}<0.05$.

ensures that inadvertently transduced non-cancerous cells will have a high probability of eventually being killed, and that the spread of the RRV will be self-limited.

Infections of MDA-MB-435 with ACE-GFP and GS4GFP at MOI 0.05 demonstrated that ACE and GS4 vectors replicated in these cells at comparable rates and eventually spread throughout entire cell populations. While ACE vector uses the Pit-2 phosphate transporter for viral entry, the GS4 vector uses the Pit-1 phosphate transporter (23). As expected, ACE-GFP and GS4-dsRed were able to co-infect MDA-MB435 cells because both of the GFP and dsRed reporter genes are expressed in the cells. Co-infections of MDA-MB-435 in vitro with GS4-yCD and ACE-TK demonstrated that dualvector suicide gene therapy could achieve better cytotoxic efficacy than single-vector suicide gene therapy.
In vivo $\mathrm{RRV}$-mediated dual-vector suicide gene therapy at MOI 0.05 efficiently suppressed MDA-MB-435 tumor growth, showing similar ability to inhibit tumors as achieved in $\mathrm{yCD}$ pretransduced tumors after prodrug administration, indicating that the high efficiency of gene transfer by replicative spread of RRV greatly augmented the efficacy of suicide gene therapy. Indeed, a higher MOI might achieve even more efficient tumor inhibition in the MDA-MB-435 tumor model; nonetheless, it should be noted that this level of initial transduction was intended to be comparable to that previously reported by injection of packaging cells producing conventional RDR vectors in clinical trials (10), and the growth suppression achieved in this study was considerably greater than would be expected from conventional vectors at such a low MOI.

Additional levels of RRV selectivity and safety may also be achieved by vector targeting through replacement of the retroviral promoter with cell-specific transcriptional elements to target viral gene expression to particular tissues $(12,24)$. This may also eventually be achieved by modification of cellbinding tropism through direct engineering of the retroviral envelope (25) or by incorporation into adenovirus-retrovirus hybrid vectors (26) with fiber modifications, thus restricting virus entry and/or replication exclusively to tumor cells, and thereby minimizing the potential risk to recipients. In conclusion, in the case of poor prognosis malignancies such as breast cancer, the use of RRVs may represent a promising experimental therapeutic strategy, particularly if the benefits justify the potential risks. With their ability to efficiently and stably transduce tumors in vivo, RRVs may achieve sufficiently persistent expression of transgenes that can achieve significant tumor inhibition and provide long-term survival benefit.

\section{Acknowledgements}

We thank Dr Noriyuki Kasahara and Dr Christopher R. Logg for generously providing the vector plasmid GS4-GFP and for many helpful suggestions and discussion. This work was supported by grants NSC95-2320-B-194-004-MY3 and NSC94-2320-B-194-007 from the National Science Council, Taiwan.

\section{References}

1. Thomas CE, Ehrhardt A and Kay MA: Progress and problems with the use of viral vectors for gene therapy. Nat Rev Genet 4: 346-358, 2003.

2. Lewis PF and Emerman M: Passage through mitosis is required for oncoretroviruses but not for the human immunodeficiency virus. J Virol 68: 510-516, 1994.

3. Miller DG, Adam MA and Miller AD: Gene transfer by retrovirus vectors occurs only in cells that are actively replicating at the time of infection. Mol Cell Biol 10: 4239-4242, 1990.

4. Varmus HE, Padgett T, Heasley S, Simon G and Bishop JM: Cellular functions are required for the synthesis and integration of avian sarcoma virus-specific DNA. Cell 11: 307-319, 1977.

5. Grote D, Russell SJ, Cornu TI, Cattaneo R, Vile R, Poland GA and Fielding AK: Live attenuated measles virus induces regression of human lymphoma xenografts in immunodeficient mice. Blood 97: 3746-3754, 2001.

6. Csatary LK, Moss RW, Beuth J, Torocsik B, Szeberenyi J and Bakacs T: Beneficial treatment of patients with advanced cancer using a newcastle disease virus vaccine (mth-68/h). Anticancer Res 19: 635-638, 1999. 
7. Gomella LG, Mastrangelo MJ, McCue PA, Maguire HC, Mulholland SG and Lattime EC: Phase I study of intravesical vaccinia virus as a vector for gene therapy of bladder cancer. J Urol 166: 1291-1295, 2001

8. Fukazawa T, Matsuoka J, Yamatsuji T, Maeda Y, Durbin ML and Naomoto Y: Adenovirus-mediated cancer gene therapy and virotherapy (Review). Int J Mol Med 25: 3-10, 2010.

9. Andreadis ST, Roth CM, Le Doux JM, Morgan JR and Yarmush ML: Large-scale processing of recombinant retroviruses for gene therapy. Biotechnol Prog 15: 1-11, 1999

10. Rainov NG: A phase iii clinical evaluation of herpes simplex virus type 1 thymidine kinase and ganciclovir gene therapy as an adjuvant to surgical resection and radiation in adults with previously untreated glioblastoma multiforme. Hum Gene Ther 11: 2389-2401, 2000

11. Gordon EM and Hall FL: The 'timely' development of Rexin-G: First targeted injectable gene vector (Review). Int J Oncol 35: 229-238, 2009

12. Logg CR, Logg A, Matusik RJ, Bochner BH and Kasahara N: Tissue-specific transcriptional targeting of a replicationcompetent retroviral vector. J Virol 76: 12783-12791, 2002.

13. Tai CK, Wang WJ, Chen TC and Kasahara N: Single-shot, multicycle suicide gene therapy by replication-competent retrovirus vectors achieves long-term survival benefit in experimental glioma. Mol Ther 12: 842-851, 2005.

14. Tai CK and Kasahara N: Replication-competent retrovirus vectors for cancer gene therapy. Front Biosci 13: 3083-3095, 2008 .

15. Nakamura H, Mullen JT, Chandrasekhar S, Pawlik TM, Yoon SS and Tanabe KK: Multimodality therapy with a replicationconditional herpes simplex virus 1 mutant that expresses yeast cytosine deaminase for intratumoral conversion of 5 fluorocytosine to 5-fluorouracil. Cancer Res 61: 5447-5452, 2001.

16. Beltinger C, Fulda S, Kammertoens T, Meyer E, Uckert W and Debatin KM: Herpes simplex virus thymidine kinase/ganciclovirinduced apoptosis involves ligand-independent death receptor aggregation and activation of caspases. Proc Natl Acad Sci USA 96: 8699-8704, 1999.
17. Freeman SM: Suicide gene therapy. Adv Exp Med Biol 465: 411-422, 2000.

18. Mesnil M and Yamasaki H: Bystander effect in herpes simplex virus-thymidine kinase/ganciclovir cancer gene therapy: Role of gap-junctional intercellular communication. Cancer Res 60: 3989-3999, 2000

19. Pan J, Zhong C, Chang Z and Roy-Burman P: A potential therapeutic strategy to combat leukemia virus infection. Cancer Biol Ther 2: 92-99, 2003.

20. Logg CR, Baranick BT, Lemp NA and Kasahara N: Adaptive evolution of a tagged chimeric gammaretrovirus: Identification of novel cis-acting elements that modulate splicing. J Mol Biol 369: 1214-1229, 2007.

21. Pear WS, Nolan GP, Scott ML and Baltimore D: Production of high-titer helper-free retroviruses by transient transfection. Proc Natl Acad Sci USA 90: 8392-8396, 1993.

22. Solly SK, Trajcevski S, Frisen C, Holzer GW, Nelson E, Clerc B, Abordo-Adesida E, Castro M, Lowenstein P and Klatzmann D: Replicative retroviral vectors for cancer gene therapy. Cancer Gene Ther 10: 30-39, 2003.

23. Overbaugh J, Miller AD and Eiden MV: Receptors and entry cofactors for retroviruses include single and multiple transmembrane-spanning proteins as well as newly described glycophosphatidylinositol-anchored and secreted proteins. Microbiol Mol Biol Rev 65: 371-389, 2001.

24. Metzl C, Mischek D, Salmons B, Gunzburg WH, Renner M and Portsmouth D: Tissue- and tumor-specific targeting of murine leukemia virus-based replication-competent retroviral vectors. J Virol 80: 7070-7078, 2006.

25. Tai CK, Logg CR, Park JM, Anderson WF, Press MF and Kasahara N: Antibody-mediated targeting of replicationcompetent retroviral vectors. Hum Gene Ther 14: 789-802, 2003.

26. Soifer H, Higo C, Logg CR, Jih LJ, Shichinohe T, HarboeSchmidt E, Mitani K and Kasahara N: A novel, helper-dependent, adenovirus-retrovirus hybrid vector: Stable transduction by a two-stage mechanism. Mol Ther 5: 599-608, 2002. 